Nota de investigación

\title{
Generación y caracterización básica de bagazos de la agroindustria del mezcal en Oaxaca
}

Paulina Alejandra Flores-Ríos ${ }^{1}$

Robles Celerino ${ }^{1 \S}$

Ernesto Castañeda-Hidalgo ${ }^{2}$

${ }^{1}$ Instituto Politécnico Nacional-CIIDIR Unidad Oaxaca. Laboratorio de Suelos, Calle Hornos 1003, Santa Cruz Xoxocotlán, Oaxaca, México. CP. 71230. Tel. 951 5170610. (floresrios-pau@hotmail.com). ${ }^{2}$ Tecnológico Nacional de México-Instituto Tecnológico del Valle de Oaxaca. Ex-Hacienda de Nazareno, Santa Cruz Xoxocotlán, Oaxaca, México. CP. 71230. Tel. 9515170444. (ernesto.ch@ voaxaca.tecnm.mx).

${ }^{\S}$ Autor para correspondencia: croblesp@ipn.mx.

\section{Resumen}

En Oaxaca, en 2019, se produjo 90.1\% del mezcal certificado producido a nivel nacional, además de una cantidad desconocida de mezcal no certificado, que en su producción generan residuos, el bagazo el de mayor importancia. Aunque en Oaxaca actualmente es subutilizado, existen diversas investigaciones sobre su potencial, que han sido destinadas a la obtención de productos y metabolitos diversos de uso industrial. Se desconoce el dato exacto de la cantidad generada en los palenques y las características de estos; sin embargo, es información importante ya que es la base para establecer estrategias de manejo. En este trabajo, realizado en la región Valles Centrales de Oaxaca durante 2017, se presenta la caracterización básica de los residuos sólidos de la agroindustria del mezcal por especie utilizada, y se estiman coeficientes que pueden ser de aplicación general para la estimación rápida de la producción de estos residuos, y sirva como base para las estrategias de manejo. Se concluye que existen diferencias en las características de los residuos y la cantidad de bagazo generado entre las diferentes especies evaluadas.

Palabras clave: Agave angustifolia, Agave karwinskii, Agave marmorata, Agave potatorum, magueyes mezcaleros, residuos agroindustriales.

Recibido: agosto de 2020

Aceptado: septiembre de 2020 
En Oaxaca, ocho de los municipios del Estado conocidos como 'Región del mezcal' están incorporados a la denominación de origen 'Mezcal' (DOF, 2018). En el estado se produjeron, en 2019, 6437680 litros de mezcal, representando 90.1\% de la producción nacional de mezcal certificado, con una clara tendencia en el aumento anual de la producción (COMERCAM, 2019) y una cantidad desconocida de mezcal sin certificar.

Actualmente, la NOM 070 (DOF, 2017) marca la clasificación de esta bebida como mezcal ancestral, mezcal artesanal y mezcal, las cuales varían en su proceso y equipos de cocimiento, molienda, fermentación y destilación, los mezcales de los tres tipos de producción se destinan predominantemente a la comercialización. En años recientes ha habido un incremento en la producción de mezcal ancestral y artesanal, así como de especies silvestres; si bien en porcentaje no se aprecia el incremento, debido a las cantidades de mezcal de agave espadín, si lo hace en la cantidad producida (COMERCAM, 2019), aumentando con ello la demanda y comercialización.

En cualquiera de los tres procesos de elaboración de mezcal se generan residuos que actualmente son de poca importancia en la agroindustria, pero con amplia repercusión ambiental. El primero de ellos son las hojas, seguidas por el bagazo y la vinaza. El bagazo de agave es el residuo fibroso que queda después de que las cabezas de agave 'piñas' son troceadas, cocidas, enjuagadas y exprimidas para extraerle los azucares fermentables para la obtención de los mostos que serán utilizados en la producción de mezcal (Íñiguez et al., 2014).

Existen diversas alternativas para el manejo del bagazo de agave que se han utilizado en la industria del tequila y proporcionan a la misma, sustentabilidad ambiental, como las que mencionan Íñiguez et al. (2014), quienes previa separación de fibra y médula, mencionan que ambas fracciones pueden destinarse a un uso potencial, la médula como parte de la dieta de rumiantes y la fibra para la elaboración de tableros conglomerados. Los mismos autores mencionan el uso de bagazo para el tratamiento de residuales de la industria de la curtiduría y de la misma industria tequilera, así como su uso con propósitos agrícolas como componente de sustratos de cultivo en diferentes sistemas; todas las anteriores con resultados exitosos y de gran potencial para el escalamiento.

En Oaxaca el bagazo aun es subutilizado, vertido en ríos, arroyos o amontonado fuera de los palenques y parcelas (Flores et al., 2013), ocasionando con esto un grave problema al ambiente debido a los grandes volúmenes generados y por sus características morfológicas, además de ser un residuo lignocelulósico de lenta degradación (Alonso y Rigal, 1997). Chávez (2010) afirma que el bagazo de agave mezcalero tiene amplio potencial y ventaja, al estar impregnado de alcoholes, al usarlo como combustible para la generación de energía renovable. Los bagazos de agave tienen amplias posibilidades de ser utilizados como sustrato hortícola alternativo o complementario a los existentes (Iñiguez et al., 2014; Zárate et al., 2014; Crespo, 2018).

Los productores de mezcal no registran la cantidad de bagazo generado en los palenques y no se han desarrollado trabajos de investigación con ese objetivo. Para bagazo de agave tequilero, Cedeño (1995) estimó que $40 \%$ del peso fresco de piña se vuelve bagazo. Algunos estudios que evalúan el potencial del bagazo de mezcal para diversos usos mencionan cifras distintas de generación de residuos, sin distinción de la especie del que provenga y sin mencionar la fuente de obtención del dato (Chávez, 2010; Íñiguez et al., 2011; Caballero et al., 2013). 
Herrera et al. (2016) indican que la evaluación de la generación y la caracterización de los residuos representan una herramienta importante para determinar las necesidades económicas, de recursos humanos y de infraestructura requeridas en las estrategias de manejo y el diseño de sistema de tratamientos que logre disminuir los impactos negativos de la industria que lo genera. El objetivo del presente trabajo es generar información sobre la producción de bagazo de cuatro especies de magueyes mezcaleros de amplio uso en Oaxaca y realizar la caracterización básica de su composición y propiedades.

El trabajo se realizó, en el año 2017, en dos palenques de los municipios Santiago Matatlán (16 51'59.3" latitud norte $96^{\circ} 22^{\prime}$ ' 43.1' longitud oeste), Santa Catarina Minas (16 46' 47.4" latitud norte $96^{\circ} 37^{\prime}$ 03.1" longitud oeste), Santa Cruz Xoxocotlán $\left(17^{\circ} 02\right.$ ' 01.3" latitud norte $96^{\circ} 43^{\prime}$ $40.5^{\prime \prime}$ longitud oeste) y San Dionisio Ocotepec (16 47'09" latitud norte 96 $16^{\circ}$ ' 40.6" longitud oeste), todos en la región Valles Centrales del Estado de Oaxaca. Los análisis fisicoquímicos se realizaron en el Laboratorio de Suelos del CIIDIR Unidad Oaxaca, en el municipio de Santa Cruz Xoxocotlán.

Se analizaron cuatro especies de agave mezcalero, Agave angustifolia Haw., Agave potatorum Zucc., Agave karwinskii Zucc. y Agave marmorata Roezl. Se registraron datos en dos procesos productivos de tipo tradicional de mezcal, por cada especie de agave. Se pesaron las piñas utilizadas en la fase de horneado, de las cuales se tomó una muestra para analizar el contenido de humedad (AOAC, 1990). Se siguió el proceso de producción hasta la obtención del residuo sólido; se registró la cantidad de litros de mezcal producidos en la primera destilación.

Al bagazo obtenido se le determinó densidad de campo (DDC) según la técnica descrita por los Test Methods for Examination of Composting and Compost (TMECC 3.01-C, 2001), se cubicó la pila para calcular su volumen y estimar posteriormente el peso producido. En cada pila de bagazo se tomaron tres muestras representativas para su análisis. Se cuantificó la proporción de fibra y médula de acuerdo a lo descrito por Iñiguez et al. (2011), se determinaron también materia seca y humedad por el método gravimétrico (AOAC, 1990), el contenido de cenizas por calcinación en horno mufla a $550{ }^{\circ} \mathrm{C}$, la proporción de masa perdida en este procedimiento se consideró materia orgánica (MO) (AOAC, 1990), el nitrógeno total (NT) se determinó con la técnica microKjeldahl (NOM-021-SEMARNAT-2000), el carbono orgánico total (COT) se calculó a partir del contenido de MO, con la ecuación de Gouleke (1977), la relación carbono/nitrógeno (C/N) se calculó con base en los contenidos de COT y NT, el pH y la conductividad eléctrica (CE) fueron medidos en una suspensión bagazo:agua con relación 1:10 peso/volumen, con un potenciómetro HANNA (CWMI, 1976).

Todas las mediciones se realizaron por triplicado. Los datos, previa comprobación de normalidad y homogeneidad de varianzas (los datos en porcentaje fueron transformados con la función Arcsen), se sometieron a un análisis de varianza y, cuando éste indicó diferencias significativas $(p \leq 0.05)$, se utilizó la prueba de Tukey para la separación de las medias entre especies de agave. Para la comparación de medias entre los tipos de molienda y destilación se aplicó una prueba de T de Student. Los datos se analizaron en el programa estadístico SPSS versión 21.0. 
La especie más utilizada y de la que se produce la proporción mayoritaria de mezcal, en los palenques muestreados y en todo el estado de Oaxaca, es $A$. angustifolia y es en ésta que se reporta el menor valor de humedad al inicio del proceso, siendo estadísticamente diferente a A. potatorum y A. marmorata (Cuadro1).

Cuadro 1. Caracterización del bagazo de cuatro especies de agave generado en la producción de Mezcal en Oaxaca, México.

\begin{tabular}{ccccc}
\hline \multirow{2}{*}{ Parámetro } & \multicolumn{4}{c}{ Especies } \\
\cline { 2 - 5 } A. angustifolia & A. karwinskii & A. potatorum & A. marmorata \\
\hline Humedad Ag (\%) & $64.59 \pm 3.7 \mathrm{~b}^{\dagger \dagger}$ & $68.92 \pm 4.78 \mathrm{~b}$ & $77.93 \pm 9.29 \mathrm{a}$ & $79.07 \pm 1.52 \mathrm{a}$ \\
Humedad Bag (\%) & $80.3 \pm 4.06 \mathrm{a}$ & $75.54 \pm 3.69 \mathrm{a}$ & $74.67 \pm 5.23 \mathrm{a}$ & $80.3 \pm 2.95 \mathrm{a}$ \\
Fibra $(\%, \mathrm{bh})$ & $29.97 \pm 6.54 \mathrm{~b}$ & $48.27 \pm 8.39 \mathrm{a}$ & $35.82 \pm 9.16 \mathrm{ab}$ & $33.93 \pm 11.35 \mathrm{~b}$ \\
Médula (\%bh) & $70.03 \pm 6.54 \mathrm{a}$ & $51.73 \pm 8.39 \mathrm{~b}$ & $64.18 \pm 9.16 \mathrm{ab}$ & $66.07 \pm 11.35 \mathrm{a}$ \\
MO (\%) & $90.21 \pm 2.65 \mathrm{a}$ & $88.4 \pm 2.88 \mathrm{a}$ & $86.57 \pm 0.97 \mathrm{a}$ & $81.84 \pm 2.58 \mathrm{~b}$ \\
$\mathrm{COT}(\%)$ & $50.12 \pm 2.16 \mathrm{a}$ & $49.11 \pm 1.6 \mathrm{a}$ & $48.19 \pm 0.44 \mathrm{a}$ & $45.46 \pm 1.44 \mathrm{~b}$ \\
$\mathrm{NT}$ & $0.26 \pm 0.1 \mathrm{a}$ & $0.22 \pm 0.05 \mathrm{a}$ & $0.26 \pm 0.06 \mathrm{a}$ & $0.31 \pm 0.1 \mathrm{a}$ \\
$\mathrm{C} / \mathrm{N}$ & $196.25 \pm 4.02 \mathrm{ab}$ & $226.03 \pm 13.13 \mathrm{a}$ & $182.33 \pm 12.27 \mathrm{~b}$ & $160.47 \pm 49.42 \mathrm{~b}$ \\
$\mathrm{pH}$ & $5.42 \pm 1.48 \mathrm{a}$ & $6.01 \pm 0.56 \mathrm{a}$ & $5.43 \pm 0.96 \mathrm{a}$ & $5.19 \pm 0.2 \mathrm{a}$ \\
$\left.\mathrm{CE}(\mathrm{dS} \mathrm{m})^{-1}\right)$ & $1.32 \pm 0.09 \mathrm{~b}$ & $1.5 \pm 0.29 \mathrm{~b}$ & $1.33 \pm 0.12 \mathrm{~b}$ & $1.92 \pm 0.35 \mathrm{a}$ \\
DDC $\left(\mathrm{kg} \mathrm{m}^{-3} \mathrm{bh}\right)$ & $657.06 \pm 60.27 \mathrm{a}$ & $589.58 \pm 49.59 \mathrm{a}$ & $536.98 \pm 89.04 \mathrm{~b}$ & $630.4 \pm 91.7 \mathrm{ab}$ \\
DDC $\left(\mathrm{kg} \mathrm{m}^{-3} \mathrm{bs}\right)$ & $138.35 \pm 5.63 \mathrm{ab}$ & $143.46 \pm 16.68 \mathrm{a}$ & $132.25 \pm 5.46 \mathrm{ab}$ & $122.771 \pm 16.83 \mathrm{~b}$ \\
\hline
\end{tabular}

$\mathrm{Ag}=$ agave (piña); $\mathrm{Bag}=$ bagazo; $\mathrm{bh}=$ base húmeda; $\mathrm{MO}=$ materia orgánica; $\mathrm{COT}=$ carbono orgánico total; $\mathrm{NT}=$ nitrógeno total; $\mathrm{C} / \mathrm{N}=$ relación carbono/nitrógeno; $\mathrm{CE}=$ conductividad eléctrica; $\mathrm{DDC}=$ densidad de campo; bs= base seca. ${ }^{\dagger}=$ letras diferentes en la misma fila indican diferencia estadística significativa (Tukey $p \leq 0.05$ ).

En las variables evaluadas en el bagazo, a excepción del contenido de NT, humedad y pH, se registran diferencias significativas entre especies. En el bagazo de las especies A. marmorata y A. angustifolia se registran mayor DDC y mayor porcentaje de humedad y médula. Íñiguez et al. (2011) reportan que el bagazo con mayor contenido de médula refleja una mejoría en las propiedades físicas cuando se composta y se usa como sustrato hortícola, mejorando los parámetros de densidad real y agua fácilmente disponible.

El bagazo de A Karwinskii presenta el menor contenido de NT y el valor más elevado de la de la relación $\mathrm{C} / \mathrm{N}$, siendo este último estadísticamente diferente al valor de otros bagazos lo que implicaría un mayor aporte de nitrógeno externo en caso de dirigirse al manejo por compostaje, ya que el valor recomendado de la relación $\mathrm{C} / \mathrm{N}$ para iniciar este proceso de manera adecuada es entre 25 y 30 (Íñiguez et al., 2011), el bagazo de las tres especies restantes tiene valores de $\mathrm{C} / \mathrm{N}$ cercanos a los reportadas por Íñiguez et al. (2011) de bagazo proveniente de dos moliendas distintas y muy por encima de los valores reportados por Crespo et al. (2018) en bagazo de agave tequilero.

El valor de $\mathrm{pH}$ del bagazo de todas las especies es superior al reportado para bagazo de A. tequilana por Íñiguez et al. (2011) y Crespo et al. (2018), pero concuerda con el valor reportado por Martínez et al. (2013) en bagazo de A. angustifolia. 
El valor de la DDC (bs) es cercano a los valores reportados por Íñiguez et al. (2011) en bagazo de agave tequilero con alto contenido en médula. La concentración de sales solubles, medida como conductividad eléctrica, es mayor y estadísticamente diferente en A. marmorata. Algunos trabajos de compostaje de bagazo reportan el incremento del valor de este parámetro en los primeros 126 a 180 días y una disminución después de los 180 (Íñiguez et al., 2011; Martínez et al., 2013). La salinidad inhibe el crecimiento de las plantas y reduce su productividad a causa de un déficit hídrico, toxicidad por los iones que la inducen y al desbalance nutrimental que ocasiona (Cepeda et al., 2014); sin embargo, un valor alto de este parámetro en compostas dirigidas a su uso como sustrato, puede ser manejado de manera satisfactoria con un programa de lixiviación controlada (Barbaro et al., 2014). El tipo de destilación afecta significativamente ( $p \leq 0.05)$ los parámetros: cenizas, COT, NT, C/N y MO (Cuadro 2).

Cuadro 2. Caracterización del bagazo de agave obtenido de dos procesos diferentes de molienda y dos procesos diferentes de destilación durante la producción de mezcal en Oaxaca, México.

\begin{tabular}{cccccc}
\hline \multirow{2}{*}{ Parámetro } & \multicolumn{2}{c}{ Destilación } & & \multicolumn{2}{c}{ Molienda } \\
\cline { 2 - 3 } \cline { 5 - 6 } & Barro & Cobre & & Tahona & Molino mecánico \\
\hline Fibra $(\%$, bh $)$ & $39.78 \pm 9.24$ & $35.85 \pm 7.22$ & & $35.18 \pm 7.91$ & $42.39 \pm 12.27$ \\
Médula $(\%$ bh) & $60.22 \pm 9.24$ & $64.15 \pm 7.22$ & & $64.84 \pm 7.91$ & $57.61 \pm 12.27$ \\
MS (\%) & $20.94 \pm 1.85$ & $24.91 \pm 3.72$ & & $22.36 \pm 3.93$ & $22.58 \pm 4.75$ \\
Cenizas $(\%)$ & $11.89 \pm 1.6$ & $15.18 \pm 3.75^{*}$ & & $14.8 \pm 2.79^{*}$ & $10.69 \pm 3.02$ \\
COT $(\%)$ & $49.21 \pm 1.47^{*}$ & $47.18 \pm 2.1$ & & $47.38 \pm 1.56$ & $49.61 \pm 1.68^{*}$ \\
NT & $0.23 \pm 0.01$ & $0.3 \pm 0.08^{*}$ & & $0.27 \pm 0.06^{*}$ & $0.23 \pm 0.08$ \\
C/N & $209.69 \pm 9.88^{*}$ & $161.41 \pm 34.28$ & & $178.85 \pm 30.15$ & $218.25 \pm 17.36^{*}$ \\
MO $(\%)$ & $88.11 \pm 1.59^{*}$ & $84.42 \pm 3.75$ & & $85.16 \pm 2.87$ & $89.31 \pm 3.02^{*}$ \\
pH & $5.76 \pm 0.57$ & $5.23 \pm 0.93$ & & $5.02 \pm 0.6$ & $6.2 \pm 0.57^{*}$ \\
CE $\left(\mathrm{mS} \mathrm{m}^{-1}\right)$ & $1465.1 \pm 195.52$ & $1406 \pm 189.75$ & & $1516.54 \pm 328.84$ & $1473.5 \pm 238.45$ \\
DDC $\left(\mathrm{kg} \mathrm{m}^{-3} \mathrm{bs}\right)$ & $133.67 \pm 7.88$ & $133.95 \pm 12.23$ & & $128.9 \pm 8.79$ & $142.65 \pm 14.05^{*}$ \\
\hline
\end{tabular}

bh= base húmeda; MS: materia seca; $\mathrm{COT}=$ carbono orgánico total; $\mathrm{NT}=$ nitrógeno total; $\mathrm{C} / \mathrm{N}=$ relación carbono/nitrógeno; $\mathrm{MO}=$ materia orgánica; $\mathrm{CE}=$ conductividad eléctrica; $\mathrm{DDC}=$ densidad de campo; $\mathrm{bs}=$ base seca; ${ }^{*}=$ diferencia estadística significativa (T-Student $p \leq 0.05$ ).

En la destilación se recuperan diversos compuestos de interés, como el etanol, pero también compuestos volátiles indeseables y compuestos de alto peso molecular que son arrastrados por vapor de agua (Escalante et al., 2012), efecto que podría suceder con compuestos nitrogenados, lo que influye en el valor de NT.

El proceso de destilación en ollas de barro con tuberías de carrizo deja el bagazo de las diversas especies de agave con menor contenido de NT, comparado con el proceso de destilación en alambiques de cobre. El bagazo obtenido de la destilación en barro muestra también mayor valor de MO y COT, en consecuencia, modifica el valor de la relación $\mathrm{C} / \mathrm{N}$, lo cual puede explicar que los bagazos obtenidos de la destilación en barro tienen un valor menor de este parámetro. El efecto sobre los valores de MO y COT podría tal vez deberse a algún proceso de transferencia de masa debida a la diferencia de concentraciones y a la interacción por calor de los materiales involucrados (Cengel, 2016). No hay estudios que hayan reportado y explicado esta diferencia. 
Por el tipo de molienda se registran diferencias significativas $(p \leq 0.05)$ en los contenidos de: cenizas, COT, C/N, MO, pH y DDC (bs). Iñiguez et al. (2011) indican que el procedimiento de trituración de las piñas de agave influye en la cantidad de fibra y médula que contiene el bagazo; los mismos autores reportan que el bagazo con mayor porcentaje de médula contiene menor COT que el bagazo con menos médula, coincidiendo con los resultados reportados en este trabajo (Cuadro 2).

El contenido de NT no es diferente estadísticamente para ambos tipos de molienda, pero sí lo es la relación $\mathrm{C} / \mathrm{N}$, debido a la variación de COT. Los valores de $\mathrm{MO}$ en bagazos derivados de la molienda en tahona son menores, comparados con los derivados de la molienda en molino mecánico. Esto podría deberse a que en la molienda por cuchillas aumenta la superficie, pero dificulta la extracción de mieles, contrario a la presión ejercida en la molienda por fricción de piedra (tahona), que no es eficiente en la generación de superficie, pero si en la extracción de mieles (Michel, 2004), lo que hace más efectivo el proceso de fermentación, ya que la molienda es un punto crítico en el proceso de elaboración del mezcal, por su influencia en el rendimiento del producto terminado (Durán y Pulido, 2007) y con ello la disminución de la materia orgánica residual. Los efectos de la molienda sobre la cantidad y composición de residuos en la agroindustria del mezcal no han sido estudiados a la fecha.

La especie más utilizada para la elaboración del mezcal es también una de las cuales se genera mayor cantidad de bagazo con relación a la materia prima (bh) (Cuadro 3).

Cuadro 3. Valores generados como indicadores para cuantificar residuos sólidos de bagazo de agave en el proceso de elaboración de mezcal en Oaxaca.

\begin{tabular}{cccccc}
\hline \multirow{2}{*}{ Coeficiente } & \multirow{2}{*}{ Unidad } & \multicolumn{4}{c}{ Especies } \\
\cline { 3 - 6 } & & A. angustifolia & A. karwinskii & A. potatorum & A. marmorata \\
\hline A: ag:bg (bh) & $(\%)$ & $75.06 \pm 23.63$ & $75.89 \pm 7.69$ & $38.41 \pm 21.35$ & $61.71 \pm 3.23$ \\
B: ag:bg (bs) & $(\%)$ & $44.82 \pm 13.98$ & $49.03 \pm 22.74$ & $42.45 \pm 6.45$ & $42.45 \pm 22.06$ \\
C: mzc:ag (bh) & $\mathrm{L} \mathrm{kg}^{-1}$ & $0.135 \pm 0.01 \mathrm{a}$ & $0.122 \pm 0.01 \mathrm{a}$ & $0.043 \pm 0 \mathrm{~b}$ & $0.058 \pm .03 \mathrm{~b}$ \\
D: mzc:ag (bs) & $\mathrm{L} \mathrm{kg}^{-1}$ & $2.63 \pm 0.45$ & $3.5 \pm 1.46$ & $5.09 \pm 2.05$ & $3.74 \pm 1.67$ \\
E: ag:mzc (bs) & $\mathrm{kg} \mathrm{L}^{-1}$ & $7.41 \pm 0.61$ & $8.22 \pm 0.68$ & $23.39 \pm 1.4$ & $20.49 \pm 11.64$ \\
F: bg:mzc (bh) & $\mathrm{kg} \mathrm{L}^{-1}$ & $5.63 \pm 2.21$ & $6.21 \pm 0.46$ & $8.85 \pm 4.46$ & $12.83 \pm 7.84$ \\
\hline
\end{tabular}

$\mathrm{A}$ y $\mathrm{B}=\mathrm{ag}: \mathrm{bg}=$ porcentaje de agave que se transforma en bagazo; $\mathrm{C}$ y D: mzc:ag= litros de mezcal obtenidos; por $1 \mathrm{~kg}$ de agave; $\mathrm{E}=$ ag:mzc: $\mathrm{kg}$ de agave necesarios para producir $1 \mathrm{~L}$ de mezcal; $\mathrm{F}=\mathrm{bg}: \mathrm{mzc}=\mathrm{kg}$ producidos de bagazo por 1 L de mezcal; bh= base húmeda; bs= base seca.

Si se analizan las especies por separado, el porcentaje de bagazo generado en bh, es diferente al reportado por Cedeño (1995) de 40\% aproximadamente de la piña fresca, excepto para $A$. potatorum, que tiene un valor cercano. Para las especies $A$. angustifolia y A. karwinskii se requieren entre $7.4 \mathrm{~kg}$ y $8.2 \mathrm{~kg}$ de agave (bh) para producir un litro de mezcal, valor menor al que reportan Chávez (2010) y Caballero et al. (2013), de 15 y $12.7 \mathrm{~kg}$, respectivamente; sin embargo, la cantidad de materia prima de A. potatorum A. marmorata, necesarios para producir un litro de mezcal es significativamente mayor que las dos primeras especies. 
Los valores de apoyo para estimar cuanto bagazo (bh) se produce, con base en la cantidad de litros de mezcal obtenidos (valor de F) no son significativamente diferentes entre las especies, sin embargo, son menores en las especies A. angustifolia, A. karwinskii y A. potatorum, que son de utilización más común que la especie A. marmorata (Cuadro 3).

Hasta el momento, el bagazo total producido en la agroindustria del mezcal en el estado de Oaxaca es difícil de estimar, por diversas causas: hay muchas micro empresas productoras que no están registradas en el Consejo Regulador de la Calidad del Mezcal (2019), por lo cual no se sabe cuánta materia prima procesan y tampoco las especies de agave que utilizan; estos residuos aun no son valorados económicamente en la cadena productiva, por el contrario, a los productores de mezcal les ocasiona gastos el deshacerse de ellos, por eso muchos optan por dejarlo amontonado fuera de sus palenques o terrenos de cultivo (Flores et al., 2013), hay escasez o ausencia de trabajos de campo relacionados a este tema.

\section{Conclusiones}

Los valores de las variables en las diferentes especies de agave evaluadas, aun cuando en su mayoría presentan diferencias significativas entre ellas, se encuentran dentro de los valores reportados por otros autores para bagazos de agave mezcalero y tequilero.

El proceso en general influye en las características de los productos obtenidos, y el mezcal tiene una amplia variedad de combinaciones en sus procesos sin perder la esencia, sin embargo, si existen diferencias en la generación de residuos según la especie utilizada.

La producción de bagazo de maguey mezcalero generado durante la producción de mezcal, así como su composición y características fisicoquímicas, varían en función de la especie de agave, el tipo de molienda y de destilación utilizadas.

\section{Agradecimientos}

La primera autora agradece la beca para estudios doctorales otorgada por el Consejo Nacional de Ciencia y Tecnología (CONACYT México) (registro 209284). El financiamiento para el desarrollo de este trabajo fue otorgado por la Secretaría de Investigación y Posgrado del Instituto Politécnico Nacional (20161141 y 20170732).

\section{Literatura citada}

Alonso, M. S. y Rigal, L. 1997. Caracterización y valorización del bagazo de Agave tequilana Weber de la industria del tequila. Rev. Chapingo, Ser. Hortic. 3(2):31-39.

AOAC. 1990. Official Methods of Analysis. Association of Official Analytical Chemists. Washington: AOAC. $115 \mathrm{p}$.

Barbaro, L. A.; Karlanian, M. y Mata, D. A. 2014. Importancia del pH y la conductividad eléctrica $(\mathrm{CE})$ en los sustratos para plantas. Ediciones INTA. Argentina. $11 \mathrm{p}$. https://inta.gob.ar/sites/default/files/script-tmp-inta_-importancia_del_ph_y_la_

conductividad_elctrica.pdf. 
Caballero, C. M.; Montes, B. J. L. y Silva R. M. E. 2013. Innovación de un molino de agave cocido para la producción de mezcal. Rev Cie Téc Agr. 22(1): 45-49.

Cedeño, C. M. 1995. Tequila production. Critical Reviews in Biotechnology. 15(1):1-11.

Çengel, Y. A. 2016. Transferencia de calor y masa. Un enfoque práctico. McGrawHill/Interamericana Editores, SA de CV, $3^{\text {ra }}$. Edición. México. Capítulo 14. 773-776 pp.

Cepeda, G. A.; Valdez, A. L. A.; Castillo, G. A. M.; Ruiz, T. N. A.; Robledo, T. V. y Mendoza, V. R. 2014. Respuestas de lechuga a la conductividad eléctrica con riego superficial y subirrigación. Rev. Mex. Cienc. Agríc. 5(7):1233-1245.

Chávez, G. L. 2010. Uso de bagazo de la industria mezcalera como materia prima para generar energía. Ingenierías. 3(47):8-47.

COMERCAM. 2019.Consejo Mexicano Regulador de la Calidad del Mezcal. Informe de actividades. http://www.crm.org.mx/pdf/inf_actividades/informe2019.pdf.

Crespo, G. M. R.; Gonzáles, E. D. R.; Rodríguez, M. R.; Ruíz, C. J. A. y Durán, P. N. 2018. Caracterización química y física del bagazo de agave tequilero comportado con biosólidos de linaza como componente de sustratos para cultivos en contenedor. Rev. Int. Contam. Ambie. 34(3):373-382. Doi: 10.20937/RICA.2018.34.03.01 http://www.scielo.org.mx/ pdf/rica/v34n3/0188-4999-rica-34-03-373.pdf.

CWMI. 1976. Cornell Waste Management Institute. The science and engineering of composting. Monitoring compost pH. http:/compost.css.cornell.edu/monitor/monitorph.html. 02/08/2008.

DOF. 2017. Diario Oficial De La Federación. Norma Oficial Mexicana NOM-070-SCFI-2016. Bebidas alcohólicas-Mezcal-Especificaciones. https://dof.gob.mx/nota_detalle.php? codigo $=5472787 \&$ fecha $=23 / 02 / 2017$.

DOF. 2018.Diario Oficial De La federación. Resolución por la que se modifica la declaración general de protección de la denominación de origen mezcal, para incluir los municipios del estado de Aguascalientes que en la misma se indican. https://www.dof.gob.mx/notadetalle.php?codigo $=5534192 \&$ fecha $=08 / 08 / 2018$.

Durán, H. M. y Pulido, J. L. 2007. Análisis de la molienda en el proceso de elaboración de mezcal. Información Tecnológica. 18(1):47-52.

Escalante, M. P.; Barba, de la R. A. P.; Santos, L. y Antonio-De León, R. A. 2012. Aspectos químicos y moleculares del proceso de producción del mezcal. BioTecnología. 16(1):57-70.

Flores, R. P. A.; Robles, P. C. y Hernández, A. 2013. Prospección de la producción de residuos en la agroindustria mezcalera de Santiago Matatlán, Oaxaca. In: Novenas Jornadas Politécnicas de Investigación en Ciencias y Tecnología. CIIDIR-IPN-Oax. CD Rom. 85 p.

Gouleke C. G. 1977. Biological prossecing: composting and hydrolysis In: handbook of solid waste maganement. Wilson, D. G (Ed.). Van Nostrand Reinhold, Nueva York. 127-225 pp.

Herrera, M. J.; Rojas, M. J. F. y Anchía, L. D. 2016. Tasas de generación y caracterización de residuos sólidos ordinarios en cuatro municipios del área metropolitana Costa Rica. Costa Rica. Universidad Nacional Heredia. Revista Geográfica de América Central. 2(57):235260.

Iñiguez, C. G.; Bernal, C. J. J.; Ramírez, M. W. and Villalvazo, N. J. 2014. Recycling agave bagasse of the tequila industry. Adv. Chem. Eng. Sci. 4(2):135-142.

Iñiguez, C. G.; Martínez, G. G.; Flores, R. P. y Virgen, G. 2011. Utilización de subproductos de la industria tequilera. Parte 9. Monitoreo de la evolución del compostaje de dos fuentes distintas de bagazo de Agave para la obtención de un substrato para jitomate. Rev. Int. Contam. Ambie. 27(1):47-59. 
Martínez, G. G. A.; Íñiguez, C. G.; Ortiz, H. Y. D.; López, J. Y. y Bautista, C. M. A. 2013. Tiempos de apilado del bagazo del maguey mezcalero y su efecto en las propiedades del compost para sustrato de tomate. Rev. Int. Contam. Ambie. 29(3):209-216.

Michel, C. C. 2004. Mejoramiento de la eficiencia en la molienda en el proceso de elaboración de mezcal potosino. Tesis de Licenciatura, Universidad Autónoma de San Luis potosí. Facultad de ingeniería. 18-33 pp.

NOM-021-SEMARNAT-2000. Norma Oficial Mexicana. 2000. Que establece las especificaciones de fertilidad, salinidad y clasificación de suelos, estudio, muestreo y análisis. http://biblioteca.semarnat.gob.mx/janium/Documentos/Ciga/libros2009/ DO2280n.pdf.

TMECC. 2001. Test methods for examination of composting and compost. http//tmecc.org/tmecc.

Zarate, N. B. H.; Morales, D. B. J. y Ortiz, G. M. 2014. Diseño de sustratos de bagazo de maguey y aplicación de normas europeas para establecer criterios de riego. Reedies. 2(2):1-12. 